\title{
Generalized heat equation and transitions between different heat-transport regimes in narrow stripes
}

\author{
A. Sellitto* \\ Dipartimento di Ingegneria Industriale, Università di Salerno, \\ Campus di Fisciano, 84084, Fisciano, Italy \\ M. Sciaccođ \\ Dipartimento di Scienze Agrarie, Alimentari e Forestali, \\ Università di Palermo, Viale delle Scienze, 90128 Palermo, Italy \\ A. Amendolat \\ Dipartimento di Ingegneria Civile, Università di Salerno, \\ Campus di Fisciano, 84084, Fisciano, Italy
}

\begin{abstract}
In the framework of weakly nonlocal thermodynamics theories, in this paper we derive a nonlocal and nonlinear heat-transport equation beyond the Fourier law by means of thermodynamic considerations in agreement with the second law. The obtained equation describes the transitions among different heat-transport regimes. The stability of the solution of that equation is also analyzed in a special case.

PACS numbers: 66.70.-f,46.05.+b,05.70.Ln. MSC: 80A17; 80A20; 74A15
\end{abstract}

Keywords: generalized heat equation, diffusive regime, phonon-hydrodynamic regime, ballistic regime

\footnotetext{
*Electronic address: ant.sellitto@gmail.com

$\dagger$ Electronic address: michele.sciacca@unipa.it

${ }^{\ddagger}$ Electronic address: adaamendola1@unisa.it
} 


\section{INTRODUCTION}

The classical Fourier law

$q_{i}=-\lambda_{i j} T_{, j}$

wherein $q_{i}$ is the local heat flux, $T$ is the temperature, and $\lambda_{i j}$ is the second-order tensor of the thermal-conductivity, has been applied in a lot of experimental works to determine the thermal conductivity of various kinds of materials, as well as to carry out useful information about the dependence of $\lambda_{i j}$ on the crystallographic direction [1]. In a solid the heat is carried by two different particles, electrons and atoms, or more precisely, the collective vibrations of atoms known as phonons [2]. In metals the majority of heat is carried by electrons, whereas the phonons are the main heat carriers in semiconductors as silicon.

Nowadays, increasingly refined measurement techniques along with Fourier law are still being used to determine the thermal conductivity of new materials on a macroscopic length scale, i.e., when the mean-free path of the heat carriers is much smaller than the characteristic size of the system in such a way that the heat-transport regime is diffusive. In the diffusive regime, phonons undergo multiple scatterings (mainly collisions of the type Umklapp phonon-phonon, phonon-impurity, phonon-defect, and secondary phonon-boundary) [3, 4].

Recent advances in material science and engineering of materials led to the production and characterization of modern materials (or devices) with characteristic structures that vary on the length scale of few nanometers. In these systems, the characteristic length becomes therefore smaller than the mean-free path of heat carriers; as a consequence, the heat-transport regime is no longer diffusive, but ballistic [5, 6]: the phonons suffer only scant internal (i.e., in the bulk of the system) scatterings, and Fourier law breaks down. Equation (1) no longer holds in the hydrodynamic phonon-transport regime [3, 4, 7], too. This is confirmed by the experimental observations on heat transfer in nanosystems which sensibly differ from the predictions of Eq. (1) [8-12. In the phonon-hydrodynamic regime the constitutive equation for the heat flux is more general than Eq. (1).

The usual main heat-transport equation for phonon hydrodynamics is the so-called GuyerKrumhansl equation [13 15]

$\tau \dot{q}_{i}+q_{i}=-\lambda \theta_{, i}+\ell^{2}\left(q_{i, j j}+2 q_{j, j i}\right)$ 
wherein $\tau$ is the relaxation time of phonons, $\theta$ is the nonequilibrium temperature [16 20], and $\ell$ is the phonon mean-free path.

Generally speaking, heat-transport equations beyond Eq. (1) are important not only from the theoretical point of view, but also for practical applications; in fact, since many nanodevices have very important commercial applications (as for example the integrated circuits used in computers which can work at very high frequencies) their thermal management represents a serious issue. That management is strictly related to the qualitative and quantitative estimate of the thermal conductivity, as well as to the understanding of the right meaning of the temperature in situations far from equilibrium. Very detailed treatments about those problems can be made by means of kinetic theory of gases [10, 21]. Useful information can be also obtained by mesoscopic approaches [22].

Although in literature one can find several theoretical models facing with the breakdown of the Fourier law at nanoscale, and describing thermal transport in nanosystems [9, 12, 2327], in this paper we aim at generalizing the Eq. (2) by means of further terms thermodynamically admissible, which may of interest both in nanosystems and superfluids and in other physical systems. Our proposal reads:

$\dot{q}_{i}=\gamma_{i j}^{0}\left(u ; q_{i} ; q_{i, j}\right) u_{, j}+\gamma_{i j}^{1}\left(u ; q_{i, j}\right) q_{j}+\alpha^{0}(u) q_{i, j j}+\alpha^{1}(u) q_{j, j_{i}}$

with

$\gamma_{i j}^{0}=\left(A_{0}+\frac{\partial A_{1}}{\partial u}+\frac{\partial A_{2}}{\partial u} q_{k, k}+\frac{\partial A_{3}}{\partial u} q_{k} q_{k}\right) \delta_{i j}+\frac{\partial A_{4}}{\partial u} q_{i} q_{j}+\frac{\partial A_{5}}{\partial u} q_{i, j}+\frac{\partial A_{6}}{\partial u} q_{j, i}$

$\gamma_{i j}^{1}=\left(A_{4} q_{k, k}+A_{7}\right) \delta_{i j}+A_{4} q_{i, j}+2 A_{3} q_{j, i}$

$\alpha^{0}=A_{5}$

$\alpha^{1}=A_{2}+A_{6}$

and $A_{i}(i=0, \ldots, 7)$ regular functions of the internal energy $u$. We are also interested to give a solution of the proposed equation and analyze its stability.

Notation 1 Throughout this paper, a superposed dot denotes the partial derivative with respect to time of the indicated argument (i.e., $\dot{f} \equiv \partial f / \partial t$ ), and the symbol, ${ }_{k}$ stands for the partial derivative with respect to the $k$-th $(k=1,2,3)$ cartesian coordinate (i.e., $f_{, k} \equiv$ $\partial f\left(\partial x_{k}\right)$. Moreover, the Einstein summation convention for repeated indices is also used. 
More in details, in Sec. II we derive a nonlinear generalization of Eq. (2); in Sec. III we show that the derived nonlinear generalization of Eq. (2) allows to describe all the possible heat-transport regimes; in Sec. IV] we study the stability of the heat flux in particular situations, and finally in Sec. $\mathrm{V}$ we summarize the main results of the present paper. At last, in order to keep the attention of the readers on the main topics, some calculations are reported in an Appendix for the sake of completeness at the end of the paper.

Notation 2 Since the arguments of our studies regards solid materials, we assume that our fields are defined in a fixed and compact subset $\Omega \subset \mathbb{R}^{3}$ with a piecewise smooth boundary $\partial \Omega$. Furthermore, we assume for $\Omega$ the mathematical model of a rigid body, with the boundary $\partial \Omega$ independent of time $t$.

\section{A WEAKLY NONLOCAL AND NONLINEAR GENERALIZATION OF THE GUYER-KRUMHANSL EQUATION}

In the present section we derive a nonlinear generalization of the Guyer-Krumhansl equation (2) in the framework of a weakly nonlocal thermodynamics theory.

Weakly nonlocal thermodynamic theories allow to set-up interesting theoretical models which can suitably describe all phenomena in which the behavior of a material in any point is strictly influenced by the thermomechanical features of its closest points [14, 28, 29]. This is just what happens, for example, at nanoscale, where not only the nonlocal effects, but also the nonlinear ones play a crucial role [7, 10, 22].

In weakly nonlocal thermodynamics the gradients of the thermodynamic variables may belong to the state space [30], too. As a consequence, in the state-space variables' governing equations higher-order spatial derivatives may appear.

Let the state space $\mathcal{Z}$ be spanned by the internal energy per unit volume $u$, the local heat flux $q_{i}$, together with their first-order gradients $u_{, i}$ and $q_{i, j}$, namely, let

$\mathcal{Z} \equiv \mathcal{Z}\left\{u ; q_{i} ; u_{, i} ; q_{i, j}\right\}$

be defined in a fixed and compact subset $\Omega \subset \mathbb{R}^{3}$ with a piecewise smooth boundary $\partial \Omega$.

According with Extended Irreversible Thermodynamics (EIT) [10, 22], all the state-space variables must have their own evolution equation. The evolution equation for $u$ directly 
follows from the local balance of energy which for $\Omega$, in the absence of external heat supply (for the sake of a formal simplicity), reads:

$\dot{u}=-q_{i, i}$

Moreover, the evolution equation of $q_{i}$ has the following general form:

$\dot{q}_{i}=\Phi_{i j, j}^{(q)}+\sigma_{i}^{(q)}$

where the second-order tensor $\Phi_{i j}^{(q)}$ is the flux of $q_{i}$, and the vector $\sigma_{i}^{(q)}$ is the source term. Both quantities have to be given by constitutive equations, that is, they have to be assigned as suitable functions of the state variables in $\mathcal{Z}$. We therefore assume:

$\Phi_{i j}^{(q)}=\left[A_{1}(u)+A_{2}(u) q_{k, k}+A_{3}(u) q_{k} q_{k}\right] \delta_{i j}+A_{4}(u) q_{i} q_{j}+A_{5}(u) q_{i, j}+A_{6}(u) q_{j, i}$

$\sigma_{i}^{(q)}=A_{0}(u) u_{, i}+A_{7}(u) q_{i}$

wherein $A_{k}(u), k=0, \ldots, 7$, are regular scalar-valued functions of $u$.

Finally, the evolution equations for $u_{, i}$ and $q_{i, j}$ arise by taking the gradient extensions of Eqs. (6) and (7).

The final proposal is then:

$\dot{u}=-q_{i, i_{1}}$

$\dot{q}_{i}=\gamma_{i j}^{0}\left(u ; q_{i} ; q_{i, j}\right) u_{, j}+\gamma_{i j}^{1}\left(u ; q_{i, j}\right) q_{j}+\alpha^{0}(u) q_{i, i_{j}}+\alpha^{1}(u) q_{j, j_{i}}$

$\dot{u}_{, i}=-q_{j,{ }_{j i}}$

$\dot{q}_{i, j}=\mathcal{F}\left(u ; q_{i} ; u_{, i} ; q_{i, j} ; u_{, i j} ; q_{i, j k s}\right)$

with the above coefficients defined by:

$\gamma_{i j}^{0}=\left(A_{0}+\frac{\partial A_{1}}{\partial u}+\frac{\partial A_{2}}{\partial u} q_{k, k}+\frac{\partial A_{3}}{\partial u} q_{k} q_{k}\right) \delta_{i j}+\frac{\partial A_{4}}{\partial u} q_{i} q_{j}+\frac{\partial A_{5}}{\partial u} q_{i, j}+\frac{\partial A_{6}}{\partial u} q_{j, i}$

$\gamma_{i j}^{1}=\left(A_{4} q_{k, k}+A_{7}\right) \delta_{i j}+A_{4} q_{i, j}+2 A_{3} q_{j, i}$

$\alpha^{0}=A_{5}$

$\alpha^{1}=A_{2}+A_{6}$ 
and $\mathcal{F}$ is a regular function of the indicated arguments:

$$
\begin{aligned}
\mathcal{F}= & \gamma_{i k}^{0} u_{, j k}+\gamma_{i k}^{1} q_{k, j}+\alpha^{0} q_{i_{j, k}}+\alpha^{1} q_{k, i j k}+\left(\frac{\partial A_{2}}{\partial u} q_{k, j k}+2 \frac{\partial A_{3}}{\partial u} q_{k} q_{k, j}\right) u_{, i} \\
& +\left[\frac{\partial A_{4}}{\partial u}\left(q_{k} q_{i, j}+q_{i} q_{k, j}\right)+\frac{\partial A_{5}}{\partial u} q_{i, j k}+\frac{\partial A_{6}}{\partial u} q_{k, i j}\right] u_{, k} \\
& +\left(\frac{\partial \gamma_{i k}^{0}}{\partial u} u_{, k}+\frac{\partial \gamma_{i k}^{1}}{\partial u} q_{k}+\frac{\partial \alpha^{0}}{\partial u} q_{i_{k k}}+\frac{\partial \alpha^{1}}{\partial u} q_{k, i k}+\frac{\partial A_{4}}{\partial u} q_{i}\right) u_{,_{j}} \\
& A_{4} q_{i} q_{k, j k}+\left(A_{4} q_{k, k}+A_{7}\right) q_{i, j}+\left(A_{4} q_{i_{, j k}}+2 A_{3} q_{k, i j}\right) q_{k}+\left(A_{4} q_{i_{, k}}+2 A_{3} q_{k, i}\right) q_{k, j}
\end{aligned}
$$

Note that in Eq. (9b) both the tensorial functions $\gamma_{i j}^{k},(k=0,1)$, and the scalar functions $\alpha^{k},(k=0,1)$, are well-defined on the state space $\mathcal{Z}$.

\section{A. Compatibility with second law of thermodynamics}

In this subsection we analyze the conditions, if any, under which all the governing equations (9) are compatible with the second law of thermodynamics. In terms of the entropy per unit volume $s$, second law reads

$\dot{s}+\Phi_{i, i}^{(s)}=\sigma^{(s)}$

with $\Phi_{i}^{(s)}$ being the entropy flux, and $\sigma^{(s)}$ the entropy production which has to be always nonnegative in any admissible thermodynamic process; thus, at the present stage the question whether Eq. $9 \mathrm{~b}$ ) is physically consistent, or not, naturally arises. We thus state the following theorem.

Theorem 1 Let the state space $\mathcal{Z}$ be satisfying the equations (9) with the entropy equation (12). If we assume that $s$ and $\Phi_{i}^{(s)}$ depend on $\left(u ; q_{i} ; u_{, i} ; q_{i, j}\right)$, namely

$$
\begin{aligned}
& s=s\left(u ; q_{i} ; u_{, i} ; q_{i, j}\right) \\
& \Phi_{i}^{(s)}=\Phi_{i}^{(s)}\left(u ; q_{i} ; u_{, i} ; q_{i, j}\right)
\end{aligned}
$$

then the evolution equations (9) are compatible with second law of thermodynamics. Furthermore, the nonequilibrium entropy s per unit volume and entropy flux take the following forms which are free from the two fields $\left(u_{, i} ; q_{i, j}\right)$ :

$s=s_{0}(u)-s_{q}(u) q_{i} q_{i}$ 
$\Phi_{i}^{(s)}=\Phi_{i 0}^{(s)}\left(u ; q_{i}\right)+s_{q}(u) \alpha^{0}(u) q_{k, k} q_{i}+s_{q}(u) \alpha^{1}(u) q_{j} q_{j, i}$

wherein $s_{0}=s_{0}(u)$ is the local-equilibrium value of the entropy per unit volume, $s_{q}=s_{q}(u)$ is a regular scalar function of the internal energy per unit volume, and $\Phi_{i 0}^{(s)}$ is a regular vectorial function of the indicated arguments with $\alpha^{k},(k=0,1)$ given by Eqs. (10c) and (10d).

Domanda: Il fatto che entropia e flusso di entropia non dipendono dai due campi $\left(u_{, i} ; q_{i, j}\right)$ non e' dovuto al fatto che noi scegliamo le loro equazioni semplicemente facendo le derivate delle equazioni per $u$ e per $q$ ? In teoria sono 4 ma in pratica 2 ?

Our goal can be reached by means of the extended Liu procedure developed in Ref. [31].

Procedure 1 An extended Liu inequality is formed by adding the entropy inequality and a linear combination of the governing equations of the wanted fields and of their gradient extensions up to the order of the spatial derivatives entering the constitutive equations. The factors multiplying the field equations and their extensions (i.e., the Lagrange multipliers) only depend on the elements of the state space. Thermodynamic restrictions on the constitutive functions are obtained by an analytical procedure which starts by the observation that this inequality is linear in the highest derivatives of the unknown fields, which are considered to be completely arbitrary [31].

Proof. The theorem is proved if we impose that the second law holds by means of the extended Liu's method of Lagrange multipliers [31] as stated in Procedure 1. We need also to investigate the thermodynamic compatibility of Eqs. (7) and (8).

Now, we explicitly apply the extended Liu inequality [31] to Eq. (12) under the assumptions in Eqs. (9) and (13). It is

$$
\begin{aligned}
& \frac{\partial s}{\partial u} \dot{u}+\frac{\partial s}{\partial q_{i}} \dot{q}_{i}+\frac{\partial s}{\partial u_{, i}} \dot{u}_{, i}+\frac{\partial s}{\partial q_{i, j}} \dot{q}_{i, j}+\frac{\partial \Phi_{i}^{(s)}}{\partial u} u_{, i}+\frac{\partial \Phi_{i}^{(s)}}{\partial q_{j}} q_{i, j}+\frac{\partial \Phi_{i}^{(s)}}{\partial u_{, j}} u_{, i j}+\frac{\partial \Phi_{i}^{(s)}}{\partial q_{j, k}} q_{i, j k} \\
& -\Lambda^{u}\left(\dot{u}+q_{i, i}\right)-\Lambda_{i}^{q}\left(\dot{q}_{i}-\gamma_{i j}^{0} u_{, j}-\gamma_{i j}^{1} q_{j}-\alpha^{0} q_{i, j j}-\alpha^{1} q_{j, j i}\right) \\
& -\Lambda_{i}^{u}\left(\dot{u}+q_{j, j i}\right)-\Lambda_{i j}^{q}\left[\dot{q}_{i, j}-\mathcal{F}\left(u ; q_{i} ; u_{, i} ; q_{i, j} ; u_{, i j} ; q_{i, j k s}\right)\right] \geq 0
\end{aligned}
$$

wherein $\Lambda^{u}, \Lambda_{i}^{q}, \Lambda_{i}^{u}$ and $\Lambda_{i j}^{q}$ are the Lagrange multipliers. Note that in the inequality (16) we write the evolution equations of $q_{i}$ and $q_{i, j}$ in a compact notation only for the sake of 
a formal simplicity. It is understood, therefore, that therein Eqs. (10) and (11) have to be taken into account.

In the inequality (16) the quantities $q_{i, j k k}$ and $q_{k, i j k}$ are highest derivatives; therefore, they can assume completely arbitrary values [31]. In order that inequality (16) is always fulfilled, then the following relation has to hold:

$$
\begin{aligned}
0 & =\Lambda_{i j}^{q}\left(\alpha^{0} q_{i, j k k}+\alpha^{1} q_{k, i j k}\right)=\Lambda_{i j}^{q}\left(\alpha^{0} q_{i_{j, k s}}+\alpha^{1} q_{k, i j s}\right) \delta_{k s} \\
& =\left(\Lambda_{i j}^{q} \alpha^{0} q_{i, j k s}+\Lambda_{j i}^{q} \alpha^{1} q_{k, j i s}\right) \delta_{k s}=\left(\Lambda_{i j}^{q} \alpha^{0} \delta_{k s}+\Lambda_{j k}^{q} \alpha^{1} \delta_{i s}\right) q_{i, j k s}
\end{aligned}
$$

A sufficient condition ensuring Eq. (17) is

$0=\Lambda_{i j}^{q} \alpha^{0} \delta_{k s}+\Lambda_{j k}^{q} \alpha^{1} \delta_{i s}=\Lambda_{i j}^{q} \alpha^{0}+\Lambda_{j k}^{q} \alpha^{1} \delta_{i k}=\Lambda_{i j}^{q} \alpha^{0}+\Lambda_{j i}^{q} \alpha^{1}$

which implies

$\Lambda_{i j}^{q}=0$

In the inequality (16) the time derivatives of the state-space variables are further highest derivatives, as well as the second-order gradient $u_{, i j}$. Therefore we also have the following set of thermodynamic restrictions:

$$
\begin{aligned}
& \left(\frac{\partial s}{\partial u}-\Lambda^{u}\right) \dot{u}=0 \Rightarrow \frac{\partial s}{\partial u}=\Lambda^{u} \\
& \left(\frac{\partial s}{\partial q_{i}}-\Lambda_{i}^{q}\right) \dot{q}_{i}=0 \Rightarrow \frac{\partial s}{\partial q_{i}}=\Lambda_{i}^{q} \\
& \left(\frac{\partial s}{\partial u_{, i}}-\Lambda_{i}^{u}\right) \dot{u}_{, i}=0 \Rightarrow \frac{\partial s}{\partial u_{, i}}=\Lambda_{i}^{u} \\
& \frac{\partial s}{\partial q_{i, j}} \dot{q}_{i, j}=0 \Rightarrow \frac{\partial s}{\partial q_{i, j}}=0 \\
& \frac{\partial \Phi_{i}^{(s)}}{\partial u_{, j}} u_{, i j}=0 \Rightarrow\left(\frac{\partial \Phi_{i}^{(s)}}{\partial u_{, j}}\right)^{\mathrm{sym}}=0
\end{aligned}
$$

once the thermodynamic restriction in Eq. (19) have been taken into account.

In the inequality (16) the quantities $q_{i,{ }_{j k}}$, instead, are higher derivatives [31]. As a consequence, in order that it is never violated, a further necessary and sufficient condition is

$$
\begin{gathered}
\frac{\partial \Phi_{i}^{(s)}}{\partial q_{j, k}} q_{i, j k}+\Lambda_{i}^{q} \alpha^{0} q_{i, j_{k}} \delta_{j k}+\left(\Lambda_{i}^{q} \alpha^{1}-\Lambda_{i}^{u}\right) q_{i, j k} \delta_{i k}=0 \Rightarrow \\
\frac{\partial \Phi_{i}^{(s)}}{\partial q_{j, k}}=-\Lambda_{i}^{q} \alpha^{0} \delta_{j k}+\left(\Lambda_{j}^{u}-\Lambda_{j}^{q} \alpha^{1}\right) \delta_{i k}
\end{gathered}
$$


The thermodynamic restrictions (20) and (21) can be exploited in order to obtain the form of $s$ and $\Phi_{i}^{(s)}$. In fact, from Eqs. 20) we can firstly have

$s=s\left(u ; q_{i} ; u_{, i}\right)$

$\Phi_{i}^{(s)}=\Phi_{i}^{(s)}\left(u ; q_{i} ; q_{i, j}\right)$

Then, by simple integrations of Eqs. 20a - 20c and Eq. (21) we have

$s=s_{0}(u)+\Lambda_{i}^{q} q_{i}+\Lambda_{i}^{u} u_{, i}$

$\Phi_{i}^{(s)}=\Phi_{i 0}^{(s)}\left(u ; q_{i}\right)-\Lambda_{i}^{q} \alpha^{0} q_{k, k}+\left(\Lambda_{j}^{u}-\Lambda_{j}^{q} \alpha^{1}\right) q_{j, i}$

Recalling that Eq. 23a has to be compatible with the principle of maximum entropy at equilibrium [32], tentatively we may assume that

$\Lambda_{i}^{q}=-s_{q}(u) q_{i}$

$\Lambda_{i}^{u}=-s_{u}(u) u_{, i}$

Indeed, in order that those relations are compatible with Eq. 22b), from Eq. 23b we have

$\Lambda_{i}^{u}=0$

Finally, the coupling of Eqs. (23)-(25) leads to Eqs. (14) and (15), and the Theorem is proved.

Equation (9b) represents a nonlinear extension of the Guyer-Krumhansl equation (2) in a weakly nonlocal thermodynamic theory, and it includes many situations of interest. Equation (9b), for example, generalizes the following models existing in literature [33]:

i. Flux limiters and effective thermal conductivity of short one-dimensional nanotube when Eq. 9b) reduces to

$q_{x}=-\lambda\left(1+\beta q_{x}^{2}\right) \theta_{, x}$

with $x$ being the sole cartesian coordinate.

ii. Thermal rectification in a troncoconical nanowire when Eq. 9b reduces to $\left[1+\left(\mu+\mu^{\prime}\right) q_{x, x}\right] q_{x}=-\lambda\left(1+\beta q_{x}^{2}\right) \theta_{, i}+\ell^{2} q_{x, x x}$ where $\mu=-2 \tau /\left(c_{v} \theta\right)$, and $\mu^{\prime}=\tau_{\mathrm{tm}} /\left(c_{v} \theta\right)$ with $c_{v}$ being the specific heat at constant volume, and $\tau_{\mathrm{tm}}$ the relaxation time in the thermomass theory [25, 34, 35]. 
iii. Radial heat transport in thin layers and anomalies in temperature profile when Eq. 9b reduces to

$$
q_{j}\left(\mu q_{j, i}+\mu^{\prime} q_{i, j}\right)+q_{i}=-\lambda \theta_{, i}+\ell^{2} q_{i_{j j}}
$$

In the next sections, instead, we focus our attention to the following special case of Eq. (9b), that to our knowledge has not been taken into account in literature:

$\tau \dot{q}_{i}+q_{i}=-\lambda \theta_{, i}+\ell^{2}\left(q_{i_{j j}}+2 q_{j, j i}\right)+\left(\frac{2 \tau}{c_{v} T}\right) q_{j} q_{j, i}$

We also show a stationary solution of equation (26) and we investigate about its stability.

In the Appendix - Part I it is shown in which situations Eq. 9b reduces to Eq. (26) which hereafter we call (nonlinear) generalized heat-transport equation.

\section{TRANSITIONS AMONG THE DIFFERENT HEAT-TRANSPORT REGIMES}

Regimes wherein the Fourier law breaks down were discovered in bulk materials in the early twentieth century [36, 37], but only at extremely low temperatures [38, 39]. Recent studies on low-dimensional materials, however, pointed out that hydrodynamic phonon transport, as well as ballistic phonon transport, can be significant in a two-dimensional material (for example a graphene strip) at significantly higher temperatures and wider temperature ranges than in bulk materials [3], too.

All the terms appearing in Eq. (26) describe different heat-transport regimes and the transitions between them. In order to emphasize these behaviour, we consider the following dimensionless form of Eq. (26):

$$
\left(\frac{\varsigma}{a}\right) \tau^{*} \dot{q}_{i}^{*}+q_{i}^{*}=-\lambda^{*} a \theta_{, i}^{*}+\left(\ell^{*} \mathrm{Kn}\right)^{2}\left(q_{i, j j}^{*}+2 q_{j, j i}^{*}\right)+\frac{\mathrm{Kn}^{2}}{a}\left(\frac{2 \tau^{*}}{3 c_{v}^{*} T^{*}}\right) q_{j}^{*} q_{j, i}^{*}
$$

wherein all dimensionless unitary quantities have been indicated by a superscript * (see Appendix - Part II for the derivation of Eq. (27). Moreover, in the equation above $a=$ $a(\mathrm{Kn})$ is a generic function of the so-called Knudsen number, that is, $\mathrm{Kn}=\ell / L$ with $L$ being a reference length (for example it may be a characteristic size of the system at hand), and $\varsigma$ is a characteristic number.

The evaluation of the reciprocal strenght of the dimensionless parameters in equation (27) lead to different regimes, as reported below . 


\section{Steady-state regime}

When the ratio $\varsigma / a \rightarrow 0$ and $\tau^{*}<\infty$, in Eq. (27) the time variations of the heat flux can be neglected. This means that in this case one may assume steady-state situations for the heat flux, even when the other state-space variables change in time.

\section{Fourier diffusive regime}

In diffusive regime the phonons undergo multiple scattering inside the stripe since their mean-free path is much smaller than the characteristic size of the system. Moreover they do not have a macroscopic drift motion [3]. Indeed, when $(\varsigma, \mathrm{Kn}) \rightarrow(0,0)$ and for unitary values of $a$, Eq. (27) reduces to

$q_{i}^{*}=-\lambda^{*} \theta_{, i}^{*}$

and the classical Fourier law is recovered. In a generic cross section the heat-flux profile has the following (diffusive) limit behavior

$q_{\mathrm{diff}, \lim }=\lambda \frac{\Delta T}{L}$

wherein $\Delta T$ is the temperature difference through the two (longitudinal) ends of the stripe, and $L$ is the length of the stripe. Note that for a two-dimensional system one has $\theta_{{ }_{i}}=$ $[\Delta T / L, 0]$.

The heat-flux profile arising from Eq. 29) is uniform: the Umklapp phonon-phonon collisions, as well as the phonon-impurity and the phonon-defect collisions (which are felt everywhere in the bulk of the system), in fact, are prevalent over the phonon-boundary scattering (which, instead, mainly occurs near the boundary of the system, that is, in the so-called Knudsen layer [4]).

\section{Hydrodynamic phonon transport}

The term "hydrodynamic phonon transport" arose from its similarity with macroscopic transport phenomena in fluids [7, 10, 22]. Besides multiple scatterings, in the hydrodynamic regime the phonons exhibit a macroscopic drift motion [3] which is the main cause of the 
so-called phonon Poiseuille flow. In particular, in steady states the heat flow shows a substantially parabolic profile in each cross section [3, 4, 7]. Thus, for a two-dimensional system one has $q_{i}=\left[q_{x}(y), 0\right]$.

Indeed, when the values of $\varsigma$ and $\mathrm{Kn}$ are moderate, but $\mathrm{Kn}^{2} / a \ll \mathrm{Kn}^{2}$, Eq. (27) reduces to the Guyer-Krumhansl equation (2) which is the usual starting point of phonon hydrodynamics. Note that Eq. (27) also introduces an effective thermal conductivity which depends on Kn, i.e., on the characteristic size of the system.

\section{Ballistic regime}

When the Knudsen number reaches high values, the phonons suffer only scant internal (i.e., in the bulk of the system) scatterings, and the ballistic heat-transport regime arises. The consequent heat-flux profile is again uniform [4].

We note that when the ratio $\mathrm{Kn}^{2} / a \gg \mathrm{Kn}$, Eq. (27) reduces to

$$
\left(\frac{\varsigma}{a}\right) \tau^{*} \dot{q}_{i}^{*}+q_{i}^{*}=-\lambda^{*} a \theta_{, i}^{*}+\frac{\mathrm{Kn}^{2}}{a}\left(\frac{2 \tau^{*}}{3 c_{v}^{*} T^{*}}\right) q_{j}^{*} q_{j, i}^{*}
$$

which represents a nonlinear extension of the Maxwell-Cattaneo equation. The influence of the nonlinear term in the right-hand side of Eq. (30) on the speed of propagation of heat pulses has been investigated in Ref. [40].

In steady states Eq. (27) further reduces in

$$
\frac{\mathrm{Kn}^{2}}{a}\left(\frac{2 \tau^{*}}{3 c_{v}^{*} T^{*}}\right) q_{j}^{*} q_{j, i}^{*}=\lambda^{*} a \theta_{, i}^{*}
$$

Since we are analyzing situations wherein $\mathrm{Kn}$ is continuously increasing, and recalling that for a two-dimensional system one has $q_{i}=\left[q_{x}(y), 0\right]$ and $\theta_{, i}=[\Delta T / L, 0]$, than it is easy matter to recover from Eq. (31) the following (ballistic) limit behavior

$q_{\mathrm{ball}, \lim }=\lambda a \frac{\Delta T}{L}$

for the heat-flux profile in a generic cross section. The heat-flux profile becomes again uniform. However, we note that the predicted form of the heat-flux profile depends on the function $a$ now (i.e., on the Knudsen number), in contrast with the heat-flux profile given by Eq. (29). In the ballistic limit a possible form of the function $a$ has been derived in Ref. [4] (see Eq. (2.8) therein). 


\section{STABILITY OF THE SOLUTIONS OF THE GENERALIZED HEAT- TRANSPORT EQUATION}

By means of the (nonlinear) generalized heat-transport equation (26), in Ref. [41] the analogy between the transport phenomena in fluids and the hydrodynamic regime was emphasized. In particular, when in Eq. (26) the heat flux can be neglected with respect to the other terms, in that reference the authors studied the stability of the heat flux in the special case of vanishingly small time-variations of the internal energy.

In the present section, assuming steady-state for $u$ in Eq. (6) we study the stability of the heat flux once the hypothesis of vanishing $q_{i}$ in Eq. 26) is removed, namely, we study the stability of heat flux when the following equations hold:

$$
\begin{aligned}
& q_{i_{i}}=0 \\
& \dot{q}_{i}+\omega q_{i}-\xi q_{j} q_{j, i}-\eta q_{i, j j}+\pi_{, i}=0
\end{aligned}
$$

In Eqs. (33), which arise from Eqs. (6) and (26), we set

$$
\begin{aligned}
\xi & =\frac{2}{c_{v} T} \\
\omega & =\tau^{-1} \\
\eta & =\frac{\ell^{2}}{\tau} \\
\pi & =\frac{\lambda \theta}{\tau}
\end{aligned}
$$

for a compact notation. We note, for our purpose, that the above constants $\xi, \omega$ and $\eta$ are positive. In the Appendix - Part III, just for the sake of completeness, it is shown in which situations Eqs. (6) and (26) reduce to Eqs. (33).

Theorem 2 Let the initial boundary value problem (IBVP) be constituted by Eqs. (33) together with the boundary values

$$
q_{i}\left(x_{j} ; t\right)=0, \forall x_{j} \in \partial \Omega, \forall t \geq 0
$$

and the initial ones

$$
q_{i}\left(x_{j} ; 0\right)=q_{i}^{0}\left(x_{j}\right), \forall x_{j} \in \Omega \backslash \partial \Omega
$$

Then its solutions are always stable whatever the perturbations in the initial values $q_{i}^{0}\left(x_{j}\right)$, $\forall x_{j} \in \Omega \backslash \partial \Omega$, are. 
Proof. Qui non dovremmo assumere qualcosa sugli spazi di funzione? Esempio $L^{2}$ o $L^{1}$ ?

Let us assume that

$Q_{i}=q_{i}\left(x_{j} ; t ; q_{i}^{0}\right)$

$\Pi=\pi\left(x_{j} ; t ; q_{i}^{0}\right)$

are the solutions of the IBVP in Eqs. (33), (35) and (36). Then, let us assume that

$\widetilde{Q}_{i}=q_{i}\left(x_{j} ; t ; \widetilde{q}_{i}^{0}\right)$

$\widetilde{\Pi}=\pi\left(x_{j} ; t ; \widetilde{q}_{i}^{0}\right)$

are the solutions of the IBVP defined by Eqs. (33), (35) and the new following initial conditions

$q_{i}\left(x_{j} ; 0\right)=\widetilde{q}_{i}^{0}\left(x_{j}\right)=q_{i}^{0}\left(x_{j}\right)+\delta q_{i}^{0}\left(x_{j}\right), \forall x_{j} \in \Omega \backslash \partial \Omega$

wherein $\delta q_{i}^{0}\left(x_{j}\right)$ is a generic perturbation.

We have stable solutions if, and only if, Eqs. (37) and (38) behave in the same way when $t \rightarrow \infty$.

This problem can be analyzed by introducing the following IBVP

$\delta q_{i, i}=0$

$\delta \dot{q}_{i}+\omega \delta q_{i}-\xi\left(q_{j} \delta q_{j, i}+\delta q_{j} q_{j, i}+\delta q_{j} \delta q_{j, i}\right)-\eta \delta q_{i, j j}+p_{, i}=0$

$\delta q_{i}\left(x_{j} ; t\right)=0, \forall x_{j} \in \partial \Omega, \forall t \geq 0$

$\delta q_{i}\left(x_{j} ; 0\right)=\delta q_{i}^{0}\left(x_{j}\right), \forall x_{j} \in \Omega$

the solution of which allows to understand how the perturbations

$\delta q_{i}=\widetilde{Q}_{i}-Q_{i}$

$p=\widetilde{\Pi}-\Pi$

evolve in time. That way, the stability we are looking for coincides with the stability of the problem in Eqs. 40] [42].

Indeed, from Eq. (40b) we have

$\int_{\Omega}\left[\delta \dot{q}_{i} \delta q_{i}+\omega \delta q_{i} \delta q_{i}-\xi\left(q_{j} \delta q_{j, i} \delta q_{i}+\delta q_{j} q_{j, i} \delta q_{i}+\delta q_{j} \delta q_{j, i} \delta q_{i}\right)-\eta \delta q_{i, j j} \delta q_{i}+p_{, i} \delta q_{i}\right] d \Omega=0$

Below, in sequence, we analyze now some terms entering Eq. 42. 


$$
\int_{\Omega} \delta \dot{q}_{i} \delta q_{i} d \Omega=\frac{d}{d t}\left(\frac{1}{2} \int_{\Omega} \delta q_{i} \delta q_{i} d \Omega\right)
$$

$$
\int_{\Omega} q_{j} \delta q_{j, i} \delta q_{i} d \Omega=\int_{\Omega}\left(q_{j} \delta q_{j} \delta q_{i}\right)_{, i} d \Omega-\int_{\Omega} q_{j, i} \delta q_{j} \delta q_{i} d \Omega-\int_{\Omega} q_{j} \delta q_{j} \delta q_{i_{i}} d \Omega
$$

Owing to Eq. 40a), the last term in the right-hand side of Eq. 44 is null. Since, by means of the divergence theorem, we also have

$\int_{\Omega}\left(q_{j} \delta q_{j} \delta q_{i}\right)_{, i} d \Omega=\oint_{d \Omega} q_{j} \delta q_{j} \delta q_{i} n_{i} d A=0$

owing to Eq. (40c), finally we have

$\int_{\Omega} q_{j} \delta q_{j, i} \delta q_{i} d \Omega=-\int_{\Omega} q_{j, i} \delta q_{j} \delta q_{i} d \Omega$

$$
\int_{\Omega} \delta q_{j} \delta q_{j, i} \delta q_{i} d \Omega=\frac{1}{2} \int_{\Omega} \delta q_{i}\left(\delta q_{j} \delta q_{j}\right)_{, i} d \Omega=\frac{1}{2} \int_{\Omega}\left(\delta q_{i} \delta q_{j} \delta q_{j}\right)_{, i} d \Omega-\frac{1}{2} \int_{\Omega} \delta q_{i, i} \delta q_{j} \delta q_{j} d \Omega
$$

Owing to Eq. 40a), the last term in the right-hand side of Eq. 46) is null. Since, by means of the divergence theorem, we also have

$\int_{\Omega}\left(q_{j} \delta q_{j} \delta q_{i}\right)_{, i} d \Omega=\oint_{d \Omega} q_{j} \delta q_{j} \delta q_{i} n_{i} d A=0$

owing to Eq. 40c), finally we have

$\int_{\Omega} \delta q_{j} \delta q_{j, i} \delta q_{i} d \Omega=0$

$$
\int_{\Omega} \delta q_{i, j j} \delta q_{i} d \Omega=\int_{\Omega}\left(\delta q_{i, j} \delta q_{i}\right)_{, j} d \Omega-\int_{\Omega} \delta q_{i, j} \delta q_{i, j} d \Omega
$$

The use again of the divergence theorem yields

$$
\int_{\Omega}\left(\delta q_{i, j} \delta q_{i}\right)_{, j} d \Omega=\oint_{d \Omega} \delta q_{i} \delta q_{i, j} n_{j} d A=0
$$

owing to Eq. (40c), and then from Eq. (48) we obtain

$$
\int_{\Omega} \delta q_{i, j j} \delta q_{i} d \Omega=-\int_{\Omega} \delta q_{i, j} \delta q_{i, j} d \Omega
$$




$$
\int_{\Omega} p_{, i} \delta q_{i} d \Omega=\int_{\Omega}\left(p \delta q_{i}\right)_{, i} d \Omega-\int_{\Omega} p \delta q_{i, i} d \Omega=\oint_{d \Omega} p \delta q_{i} n_{i} d A=0
$$

once Eqs. 40a and 40c have been taken into account.

When Eqs. (43), (45), (47), (49) and (50) are inserted into Eq. (42) we have

$\frac{d}{d t}\left(\frac{1}{2} \int_{\Omega} \delta q_{i} \delta q_{i} d \Omega\right)=-\omega \int_{\Omega} \delta q_{i} \delta q_{i} d \Omega-\eta \int_{\Omega} \delta q_{i, j} \delta q_{i, j} d \Omega \leq-(\omega+P \eta) \int_{\Omega} \delta q_{i} \delta q_{i} d \Omega$

due to the Poincaré inequality, the positive constant value $P$ depending on $\Omega$.

Now, if we define the average energy of the disturbance as

$\mathcal{E}(t)=\frac{1}{\mathcal{M}(\Omega)} \int_{\Omega} \delta q_{i} \delta q_{i} d \Omega$

being $\mathcal{M}(\Omega)$ the measure of the volume of $\Omega$, from inequality $(51)$ the following ordinary differential equation arises

$\dot{\mathcal{E}} \leq-2(\omega+P \eta) \mathcal{E} \Rightarrow \mathcal{E}(t) \leq \mathcal{E}(0) e^{-2(\omega+P \eta) t}$

with $\mathcal{E}(0)$ being the initial value of the average energy of the disturbance. Since

$\lim _{t \rightarrow \infty} \frac{\mathcal{E}(t)}{\mathcal{E}(0)}=0$

the solution of Eqs. (40) are stable to the initial perturbations, and the theorem is proved.

\section{FINAL COMMENTS}

Material systems with characteristic sizes of the order of nanometers exhibit interesting optical, magnetic, electrical, and/or photoelectrical properties. In particular, the analysis of heat transfer in two-dimensional situations has become a topic of current interest since systems as silicon nanolayers, boron nitride nanolayers and graphene stripes have especially attracted the attention of several research groups for their unique features. Depending on the operating hypotheses (in particular the temperature range, and the characteristic size) the transport of phonons, which are the dominant heat carriers in non-metallic solids, may change. Phonons, in fact, can undergo not only a diffusive regime, but also a hydrodynamic 
(Poiseuille-like) regime and a ballistic one [3 [5, 43]. In the first case, heat transfer is describable by means of Fourier law. In the latter cases, instead, Eq. (1) breaks down and more general constitutive equations for the local heat flux are needed. Different theories and/or approaches can be found in literature to face with the breakdown of Fourier law at nanoscale and to describe thermal transport in nanosystems [9, 12, 23, 27].

By means of pure theoretical considerations in agreement with well-known tenets of continuum mechanics, in this paper we derived a generalized heat equation, namely, Eq. 9b and we have shown that its simplified version (26) is able to describe (depending on the particular value of the Knudsen number) the transitions from the different heat-transport regimes. We also study the stability of the solution of Eq. 26) in a special situation, i.e., when the heat flux vector is solenoidal.

At the very end, it seems worth noticing that in present analysis no special attention has been put on the interactions between phonons and boundaries which, instead, may play a very relevant role, as shown in Refs. [4, 7, 22] for example. In those works, in fact, the phonon-boundary interactions have been modeled by introducing the following first-order no-slip boundary condition

$$
\frac{\partial q_{i}\left(x_{j} ; t\right)}{\partial \mathbf{n}}=\frac{q_{i}\left(x_{j} ; t\right)}{C \ell}, \forall x_{j} \in \partial \Omega, \forall t \geq 0
$$

with $\mathbf{n}$ being the outward normal direction to the boundary, and $C$ a suitable constant nondimensional parameter which depends on the features of the boundary. The coupling of Eq. (26) with Eq. (55) for the phonon-boundary interactions, which involves to re-investigate the stability of the heat flux, can turn out more exhaustive models for the understanding of thermal transport at nanoscale.

\section{Acknowledgements}

Work performed under the auspices of the Italian National Group of Mathematical Physics (GNFM-INdAM) which supported the present research by means of "Progetto Giovani 2018/Heat-pulse propagation in FGMs"

A. S. acknowledges the University of Salerno for the financial supports under grant no. 300393FRB17CIARL and grant "Fondo per il finanziamento iniziale dell'attività di ricerca". 


\section{Appendix}

\section{Part I}

In Sec. II we claimed that Eq. (26) is only a special case of Eq. (9b). Here we show in which case this is true. To do this, we firstly recall that in the framework of EIT [10, 22] one has

$\frac{1}{\theta}=\frac{\partial s}{\partial u}$

namely, the Lagrange multipliers $\Lambda^{u}$ in Eq. 20a coincides with the reciprocal of the nonequilibrium temperature. Therefore, once Eq. (14) is inserted into Eq. (56), one has

$\frac{1}{\theta}=\frac{1}{T}-\left(\frac{\partial s_{q}}{\partial u}\right) q_{i} q_{i}$

The second term in the right-hand side of Eq. (57), which represents the corrective term due to nonequilibrium situations, is usually very small [10] and therefore here we neglect it, namely, we assume

$\frac{1}{\theta} \approx \frac{1}{T}$

Now, if in Eqs. (10) we set

$$
\begin{aligned}
& A_{0}+\frac{\partial A_{1}}{\partial u}=-\frac{\lambda}{\tau c_{v}} \\
& A_{2}=A_{5}=A_{6}=\frac{\ell^{2}}{\tau} \\
& A_{3}=\frac{1}{\tau \theta} \\
& A_{4}=0
\end{aligned}
$$

from Eq. (9b) one obtains Eq. 26) whenever the phonon mean-free path, the relaxation time and the specific heat at constant volume are assumed constant (i.e., their variations with the temperature are negligibly small), the relations $c_{v} d \theta=d u$ and the approximation in Eq. (58) have been taken into account. Note that if $T$ means the local equilibrium temperature, $\partial A_{3} / \partial u=0$. 


\section{Part II}

Here we derive Eq. (27). To do it, in Eq. (26) we express each quantity as the product of the corresponding reference quantity (indicated by a superscript $r$ ) and the nondimensional unitary quantity (indicated by a superscript ${ }^{*}$ ). In this way, from the nonlinear heat-transport equation (26) we have

$\left(\frac{\tau^{r} q^{r}}{t^{r}}\right) \tau^{*} \dot{q}_{i}^{*}+q^{r} q_{i}^{*}=-\left(\frac{\lambda^{r} T^{r}}{L^{r}}\right) \lambda^{*} \theta_{, i}^{*}+\left(\frac{\ell^{r 2} q^{r}}{L^{r 2}}\right) \ell^{* 2}\left(q_{i, j j}^{*}+2 q_{j, j i}^{*}\right)+\left(\frac{2 \tau^{r} q^{r 2}}{c_{v}^{r} T^{r} L^{r}}\right)\left(\frac{\tau^{*}}{c_{v}^{*} T^{*}}\right) q_{j}^{*} q_{j, i}^{*}$

Since the right choice of the reference quantities plays a very important role, we observe that they may be the standard values of the physical quantities at which the system works, for example. Then, setting

$$
\begin{aligned}
& \lambda^{r}=\frac{c_{v}^{r} \ell^{r 2}}{3 \tau^{r}} \\
& t^{r}=\frac{c_{v}^{r} L^{r 2}}{\lambda^{r}} \\
& q^{r}=\frac{a \lambda^{r} T^{r}}{L^{r}} \\
& \varsigma=\frac{\tau^{r} q^{r}}{c_{v}^{r} T^{r} L^{r}}
\end{aligned}
$$

after some algebra Eq. 27) arises from Eq. 60 if the reference mean-free path $\ell^{r}$ coincides with the effective mean-free path $\ell$ (i.e., it evaluated at the reference temperature $T^{r}$ ), and $L^{r}$ coincides with the characteristic size of the system. Note that for the identification in Eq. (61a) we used the usual Ziman limit for the thermal conductivity [2].

\section{Part III}

Here we recall some results obtained in Ref. [41] in order to show in which non-steady state situations the heat flux can be essentially considered as a solenoidal vector. To this aim, taking into account the relation $d u=c_{v} d \theta$, let us rewrite Eq. (6) as

$\frac{c_{v}^{r} T^{r}}{t^{r}} c_{v}^{*} \dot{\theta}^{*}+\frac{q^{r}}{L^{r}} q_{i, i}^{*}=\dot{u}^{*}+a q_{i, i}^{*}=0$

with $c_{v}^{*} \theta^{*}=u^{*}$. In deriving Eq. 62 the use of Eqs. (61b) has been made. 
From Eqs. (62) one may conclude that for regular solutions of the field equations (i.e., for finite values of $\dot{u}^{*}$ ) in the evolution equation of the internal energy it is possible to neglect the term in $\dot{u}^{*}$ whenever $a \gg 1$. Therefore, in this situations, from Eq. (6) one has Eq. (33a). When Eq. (33a) holds, from Eq. (26) one also has Eq. (33b) under the assumptions in Eqs. (34. 
[1] T. N. Narasimhan, "Thermal conductivity through the 19th century," Phys. Today, vol. 63, pp. 36-41, 2010.

[2] J. M. Ziman, Electrons and Phonons. Oxford: Oxford University Press, 2001.

[3] S. Lee, D. Broido, K. Esfarjani, and G. Chen, "Hydrodynamic phonon transport in suspended graphene," Nature Comm., vol. 6, p. 6290 (9 pages), 2015.

[4] A. Sellitto, I. Carlomagno, and D. Jou, "Two-dimensional phonon hydrodynamics in narrow strips," Proc. R. Soc. A, vol. 471, p. 20150376 (13 pages), 2015.

[5] F. X. Alvarez and D. Jou, "Memory and nonlocal effects in heat transports: From diffusive to ballistic regime," Appl. Phys. Lett., vol. 90, p. 083109 (3 pages), 2007.

[6] T.-K. Hsiao, H.-K. Chang, S.-C. Liou, M.-W. Chu, S.-C. Lee, and C.-W. Chang, "Observation of room-temperature ballistic thermal conduction persisting over $8.3 \mu \mathrm{m}$ in SiGe nanowires," Nat. Nanotechnol., vol. 8, pp. 534-538, 2013.

[7] A. Sellitto, F. X. Alvarez, and D. Jou, "Geometrical dependence of thermal conductivity in elliptical and rectangular nanowires," Int. J. Heat Mass Transfer, vol. 55, pp. 3114-3120, 2012 .

[8] D. C. Cahill, W. K. Ford, K. E. Goodson, G. D. Mahan, A. Majumdar, H. J. Maris, R. Merlin, and S. R. Phillpot, "Nanoscale thermal transport," J. Appl. Phys., vol. 93, no. 2, pp. 793-818, 2003.

[9] Z. M. Zhang, Nano/Microscale Heat Transfer. New York: McGraw-Hill, 2007.

[10] D. Jou, J. Casas-Vázquez, and G. Lebon, Extended Irreversible Thermodynamics. Berlin: Springer, fourth revised ed., 2010.

[11] D. Y. Tzou, Macro- to Microscale Heat Transfer: The Lagging Behaviour. United Kingdom: Wiley, second ed., 2014.

[12] D. G. Cahill, P. V. Braun, G. Chen, D. R. Clarke, S. Fan, K. E. Goodson, P. Keblinski, W. P. King, G. D. Mahan, A. Majumdar, H. J. Maris, S. R. Phillpot, E. Pop, and L. Shi, "Nanoscale thermal transport. II. 2003-2012," Appl. Phys. Rev., vol. 1, no. 1, p. 011305 (46 pages), 2014.

[13] R. A. Guyer and J. A. Krumhansl, "Solution of the linearized phonon Boltzmann equation," Phys. Rev., vol. 148, pp. 766-778, 1966.

[14] P. Ván, "Weakly nonlocal irreversible thermodynamics - the Guyer-Krumhansl and the Cahn- 
Hilliard equations," Phys. Lett. A, vol. 290, pp. 88-92, 2001.

[15] R. Kovács and P. Ván, "Generalized heat conduction in heat pulse experiments," Int. J. Heat Mass Transfer, vol. 83, pp. 613-620, 2015.

[16] R. Luzzi, A. R. Vasconcellos, J. Casas-Vázquez, and D. Jou, "Characterization and measurement of a nonequilibrium temperature-like variable in irreversible thermodynamics," Physica A, vol. 234, pp. 699-714, 1997.

[17] D. Jou and J. Casas-Vázquez, "Nonequilibrium absolute temperature, thermal waves and phonon hydrodynamics," Physica A, vol. 163, pp. 47-58, 1990.

[18] J. Casas-Vázquez and D. Jou, "Temperature in nonequilibrium states: a review of open problems and current proposals," Rep. Progr. Phys., vol. 66, pp. 1937-2023, 2003.

[19] D. Jou, V. A. Cimmelli, and A. Sellitto, "Nonequilibrium temperatures and second-sound propagation along nanowires and thin layers," Phys. Lett. A, vol. 373, pp. 4386-4392, 2009.

[20] D. Jou and L. Restuccia, "Caloric and entropic temperatures in non-equilibrium steady states," Physica A, vol. 460, pp. 246-253, 2016.

[21] M. J. Fryer and H. Struchtrup, "Moment model and boundary conditions for energy transport in the phonon gas," Cont. Mech. Thermodyn., vol. 26, pp. 593-618, 2014.

[22] A. Sellitto, V. A. Cimmelli, and D. Jou, Mesoscopic Theories of Heat Transport in Nanosystems, vol. 6 of SEMA-SIMAI Springer Series. Springer International Publishing, 2016.

[23] G. Chen, "Ballistic-Diffusion Equations for Transient Heat Conduction From Nano to Macroscales," J. Heat Transfer - T. ASME, vol. 124, pp. 320-328, 2001.

[24] M. Grmela, G. Lebon, P. C. Dauby, and M. Bousmina, "Ballistic-diffusive heat conduction at nanoscale: GENERIC approach," Phys. Lett. A, vol. 339, pp. 237-245, 2005.

[25] D. Y. Tzou and Z. Y. Guo, "Nonlocal behavior in thermal lagging," Int. J. Thermal Sci., vol. 49, pp. 1133-1137, 2010.

[26] M. Wang, B.-Y. Cao, and Z.-Y. Guo, "General heat conduction equations based on the thermomass theory," Frontiers Heat Mass Transfer, vol. 1, p. 013004 (8 pages), 2010.

[27] G. Lebon, H. Machrafi, M. Grmela, and C. Dubois, "An extended thermodynamic model of transient heat conduction at sub-continuum scales," Proc. R. Soc. A, vol. 467, pp. 3241-3256, 2011.

[28] P. Ván, "Weakly nonlocal irreversible thermodynamics," Ann. Phys., vol. 12, pp. 146-173, 2003. 
[29] P. Ván and T. Fülöp, "Universality in heat conduction theory: weakly nonlocal thermodynamics," Ann. Phys., vol. 524, pp. 470-478, 2012.

[30] G. Lebon, D. Jou, J. Casas-Vázquez, and W. Muschik, "Weakly nonlocal and nonlinear heat transport in rigid solids," J. Non-Equilib. Thermodyn., vol. 23, pp. 176-191, 1998.

[31] V. A. Cimmelli, A. Sellitto, and V. Triani, "A new thermodynamic framework for second-grade Korteweg-type viscous fluids," J. Math. Phys., vol. 50, p. 053101 (16 pages), 2009.

[32] J. Verhás, Thermodynamics and Rheology. Dordrecht: Kluwer Academic Publisher, 1997.

[33] A. Sellitto, V. A. Cimmelli, and D. Jou, "Analysis of three nonlinear effects in a continuum approach to heat transport in nanosystems," Physica D, vol. 241, pp. 1344-1350, 2012.

[34] B. Y. Cao and Z. Y. Guo, "Equation of motion of a phonon gas and non-fourier heat conduction," J. Appl. Phys., vol. 102, p. 053503 (6 pages), 2007.

[35] Y. Dong, Dynamical Analysis of Non-Fourier Heat Conduction and Its Application in Nanosystems. New York: Springer-Verlag Berlin Heidelberg, 2016.

[36] H. B. G. Casimir, "Note on the conduction of heat in crystals," Physica, vol. 5, pp. 495-500, 1938.

[37] J. C. Ward and J. Wilks, "The velocity of second sound in liquid helim near the absolute zero," Phil. Mag., vol. 42, pp. 314-316, 1951.

[38] C. C. Ackerman, B. Bertman, H. A. Fairbank, and R. A. Guyer, "Second sound in solid helium," Phys. Rev. Lett., vol. 16, pp. 789-791, 1966.

[39] C. C. Ackerman and R. A. Guyer, "Temperature pulses in dielectric solids," Annals of Physics, vol. 50, pp. 128-185, 1968.

[40] A. Sellitto, V. Tibullo, and Y. Dong, "Nonlinear heat-transport equation beyond fourier law: Application to heat-wave propagation in isotropic thin layers," Continuum Mech. Thermodyn., vol. 29, pp. 411-428, 2017.

[41] V. A. Cimmelli, A. Sellitto, and D. Jou, "Nonlinear evolution and stability of the heat flow in nanosystems: Beyond linear phonon hydrodynamics," Phys. Rev. B, vol. 82, p. 184302 (9 pages), 2010.

[42] D. D. Joseph, Stability of fluid motion. New York: Springer-Verlag, 1976.

[43] R. A. Guyer and J. A. Krumhansl, "Thermal conductivity, second sound and phonon hydrodynamic phenomena in nonmetallic crystals," Phys. Rev., vol. 148, pp. 778-788, 1966. 